\title{
Optimal Box Size and Mask Diameter based on Defocus Distribution for Single Particle Cryo-EM
}

\author{
T Moriya $^{1}$ \\ ${ }^{1}$ High Energy Accelerator Research Organization (KEK) \\ toshio.moriya@kek.jp
}

Recently, it has been demonstrated that single particle analysis (SPA) using $200 \mathrm{keV}$ CryoEM paired with direct electron detector (DED) is capable to reconstruct $<200 \mathrm{kDa}$ protein structures at resolution higher than $3.0 \AA$ A. However, the majority of near-atomic resolution cryoEM structures has been determined using $300 \mathrm{keV}$ cryoEMs equipped with DEDs. As a consequence, many of typical parameter settings for cryoEM session and image processing steps are based on the accumulated experience of $300 \mathrm{keV}$ cryoEMs, such as defocus range for EM sessions and amplitude contrast for CTF estimation. Therefore, we revised the parameters for $200 \mathrm{keV}$ acceleration voltage, and found out merely optimizing mask diameter and box size based on defocus distribution of dataset can improve the resolution.

Acta Cryst. (2020). A76, a189 